\title{
DESAGREGACIÓN DEL PELIGRO SÍSMICO PARA ALGUNOS SITIOS SELECCIONADOS DE MÉXICO
}

\author{
Adrián Pozos-Estrada ${ }^{(1)}$, Roberto Gómez ${ }^{(1)}$ y H.P. Hong ${ }^{(2)}$
}

\begin{abstract}
RESUMEN
Describimos la desagregación del peligro sísmico con base en un modelo aplicable a una región de la costa del Pacífico Mexicano, tomando en cuenta las zonas de fuentes sísmicas, el modelo de recurrencia, y relaciones de atenuación. También desarrollamos relaciones de atenuación aplicables a la ciudad de México (i.e., la estación de registro de CU) y las integramos en el modelo de peligro sísmico adoptado. Proveemos mapas de peligro sísmico para la región de la costa del Pacífico, y espectros de peligro uniforme para algunos sitios seleccionados, incluyendo la estación CU; notamos que las ordenadas del espectro de diseño para la Ciudad de México son mayores a los valores del espectro de peligro uniforme para un periodo de retorno de 125 años. Los escenarios sísmicos identificados muestran que, en ocasiones, la contribución al peligro sísmico para un periodo de retorno de 125 o 475 años es dominada por un solo evento y en otros casos el peligro sísmico es debido a diferentes escenarios sísmicos y tipo de temblores; en particular, para la estación CU, se observa que para un periodo de vibrar menor que aproximadamente 0.7 (s) el peligro sísmico es dominado por sismos intraslab y que los sismos interplaca dominan el peligro sísmico para periodos de vibrar mayores que éste. Para la costa del Pacífico, el peligro sísmico es dominado por sismos interplaca, y por sismos intraslab e interplaca para zonas alejadas de la costa.
\end{abstract}

Palabras clave: desagregación sísmica; espectros de peligro uniforme; sismos interplaca; sismos intraslab

\section{SEISMIC HAZARD DEAGGREGATION FOR SOME SELECTED SITES OF MEXICO}

\begin{abstract}
We describe the deaggregation of seismic hazard based on a model applicable to a region of the Pacific coast of Mexico, taking into account the seismic source zones, the recurrence model, and the attenuation relations. We also developed attenuation relations for Mexico City (i.e., the recording station of $\mathrm{CU}$ ) and integrated them in the adopted seismic hazard model. We provide contour maps of the seismic hazard for the Pacific coast of Mexico, and uniform hazard spectra for some selected sites, including the CU station; we noted that the ordinates of the design spectrum for Mexico City are greater than those for the uniform hazard spectrum for a return period of 125 years. The seismic

Artículo recibido el 15 de abril de 2014 y aprobado para su publicación el 27 de octubre de 2014. Se aceptarán comentarios y/o discusiones hasta cinco meses después de su publicación.

(1) Investigador, Instituto de Ingeniería, Universidad Nacional Autónoma de México, Coyoacán, C.P. 04510, México, D.F. APozosE@iingen.unam.mx RGomezM@iingen.unam.mx

(2) Profesor Departamento de Ingeniería Civil y Ambiental, The University of Western Ontario, London, Ontario, Canada, N6A 5B9. hongh@eng.uwo.ca
\end{abstract}


scenarios identified show that, on occasion, the contribution to the seismic hazard for a return period of 125 or 475 years is dominated by a single event and in other cases the seismic hazard is due to different seismic scenarios and earthquake types; in particular for the CU station, it is observed that for a vibration period less than about 0.7 (s) the seismic hazard is dominated for inslab events and that interplate events dominate the seismic hazard for vibration periods greater than that. For the Pacific coast, the seismic hazard is dominated by interplate earthquakes, and by intraslab and interplate events for zones away from the coast.

Keywords: seismic deaggregation; uniform hazard spectra; interplate earthquakes; inslab earthquakes

\section{INTRODUCCIÓN}

La desagregación del peligro sísmico es una forma eficiente de identificar combinaciones de valores de parámetros tales como la magnitud y la distancia de la fuente al sitio de interés, que contribuyen a un nivel de peligro sísmico específico (McGuire1995; Chapman 1995; Bazzurro y Cornell 1999; Harmsen et al. 1999). El peligro sísmico puede expresarse en términos del espectro de respuesta. Los eventos identificados que están asociados con altas frecuencias pueden ser empleados como escenarios para evaluar estructuras ingenieriles e infraestructura nueva y existente. Las características de los escenarios también pueden ser considerados para seleccionar registros del movimiento del terreno que se necesitan para analizar estructuras y verificar el diseño sísmico. Una aplicación de esto fue hecha por Reinoso y Jaimes (2009) para obtener registros sísmicos del movimiento del terreno que son compatibles con el espectro de diseño para la ciudad de México.

En lugar de considerar la desagregación basada en el espectro de respuesta, Baker et al. (2005) consideraron la desagregación del peligro sísmico en términos de la distorsión máxima de entrepiso. Hong y Goda (2006) argumentaron que debido a que las estructuras son diseñadas y construidas para proveer servicio en lugar de sólo sostener movimientos sísmicos, la desagregación del riesgo sísmico podría ser igual o más importante que aquella del peligro sísmico. Ellos aplicaron el concepto de desagregación sísmica a algunos sitios de Canadá, donde el riesgo sísmico es expresado como el costo esperado del ciclo de vida, el cual depende de la probabilidad de la fluencia incipiente y el colapso incipiente. Sin embargo, una función del costo del ciclo de vida no está disponible para la construcción mexicana, excepto la dada por Rosenblueth y Jara (1991), la cual fue empleada por otros para seleccionar el diseño óptimo (GarcíaPérez et al. 2005; Pozos-Estrada et al. 2008). Aunque las reglas de demanda de ductilidad necesarias considerando sismos interplaca e intraslab para la desagregación del riesgo sísmico están disponibles, desafortunadamente, nuestra experiencia con la función de costo dada por Rosenblueth y Jara (1991) indica que esta expresión tiene un comportamiento no realista conforme el estado de daño estructural se acerca al colapso. Por lo tanto, esta función no es considerada y la desagregación del riesgo sísmico no se lleva a cabo en el presente trabajo.

El principal objetivo de este estudio es aplicar los métodos de desagregación del peligro sísmico para identificar eventos o escenarios frecuentes que producen un nivel de peligro predefinido para varios sitios seleccionados de México. Los análisis se llevan a cabo considerando diferentes zonas de fuente sísmicas y tipos de sismos. Para los análisis, se considera un modelo de peligro sísmico que es ensamblado por García-Soto et al. (2012) para estimar espectros de peligro uniforme (EPU) y desarrollar mapas de contorno para una región de México. Este modelo de peligro sísmico toma en cuenta: a) las zonas de fuentes sísmicas y el modelo de recurrencia descrito en Ordaz y Reyes (1999) y Jaimes y Reinoso (2006), y b) coeficientes de relaciones de atenuación (o ecuaciones de predicción de movimiento fuerte), las 
cuales son desarrolladas con base en la media geométrica de las respuestas en dos direcciones ortogonales horizontales dadas por Pozos-Estrada et al. (2008) y Hong et al. (2009b). El uso de la relación de atenuación con base en la media geométrica es justificado ya que el término de error en la relación de atenuación es aplicable para una orientación aleatoria. Como las relaciones de atenuación basadas en la media geométrica y aplicables para la Ciudad de México (i.e., las estaciones de registro de Ciudad Universitaria, las cuales serán referidas como la estación CU por sencillez) no están disponibles para sismos interplaca e intraslab, incluyendo sismos más recientes, éstas son desarrollados e integradas al modelo en este estudio. Los resultados de la desagregación basados en el modelo de peligro sísmico adoptado y sus implicaciones para el diseño sísmico y la planeación para emergencia son discutidos.

\section{MODELO DE PELIGRO SÍSMICO Y DESAGREGACIÓN}

\section{Modelo de peligro sísmico}

La estimación del peligro sísmico requiere información de sismicidad definida por la caracterización de modelos de ocurrencia de los sismos, las zonas sismogénicas (fuentes sísmicas) y sus relaciones de magnitud-recurrencia correspondientes, y leyes o relaciones de atenuación (o ecuaciones de predicción de movimiento fuerte). Dicha información se puede inferir a través de catálogos y registros de movimiento del terreno históricos.

La ocurrencia de un sismo es comúnmente considerada estadísticamente homogénea en magnitud y en ubicación dentro de una zona de la fuente sísmica. La ocurrencia de un sismo en sitios de México es comúnmente modelada como un proceso Poisoniano (Esteva 1968), aunque procesos no Poisonianos han sido también considerados en la literatura (Singh et al. 1983; Hong y Rosenblueth 1988; Rosenblueth y Jara 1991; Pozos-Estrada et al. 2008). La definición de las zonas sismogénicas para regiones sísmicas activas se puede llevar a cabo basada en la actividad sísmica histórica e incorporando características geológicas y tectónicas. Para cada zona sismogénica, la magnitud de los sismos es tratada como una variable aleatoria. Es común considerar que las magnitudes siguen la relación magnitud-recurrencia de Gutenberg-Richter (i.e., distribuciones exponencial o exponencial truncada). Para algunas zonas sismogénicas en México, otros modelos probabilísticos han sido también considerados (Rosenblueth y Ordaz 1987; Ordaz y Reyes 1999) debido a la ocurrencia de sismos característicos (Singh et al. 1983). El empleo de zonas sismogénicas homogéneas con fronteras definidas es comúnmente empleado y es considerado en el presente estudio también. Esto puede ser justificado al considerar que la mayoría de las zonas sismogénicas consideradas son zonas sismogénicas altamente activas y dominadas por fallas bien caracterizadas en México (García 2006).

El modelo de peligro sísmico adoptado es el mismo modelo considerado por García-Soto et al. (2012), excepto que una relación de atenuación adicional para eventos intraslab es considerada para la Ciudad de México, así como la relación de atenuación para eventos interplaca considerando eventos sísmicos más recientes. El modelo considera las fuentes sísmicas mostradas en la figura 1, en donde las fuentes símicas son separadas en tres grupos, dependiendo del tipo de sismo y magnitud considerados. Los dos primeros grupos, ubicados cerca de la costa del Pacífico Mexicano, están asociados con sismos interplaca. El primero de ellos considera una fuente sísmica definida por 4 polígonos, los cuales abarcan una zona de subducción acotada aproximadamente por los estados de Jalisco y Chiapas. Las magnitudes de los sismos dentro de esta zona sísmica se consideran inferiores a 7 (Ordaz y Reyes 1999). El segundo grupo considera una fuente sísmica conformada por 14 polígonos con extensión similar a la primera zona definida. De acuerdo con Ordaz y Reyes (1999), las magnitudes de los sismos dentro de esta zona sísmica se consideran mayores a 7. El tercer grupo, que considera los sismos intraslab, tiene una fuente sísmica definida por dos grandes polígonos ubicados dentro de la placa oceánica subducida (Jaimes y Reinoso 
2006), (ver también Nishenko y Singh 1987; Reinoso y Jaimes 2009). Parte de esta zona sísmica se ubica por debajo del Distrito Federal.

Además de las fuentes sísmicas anteriormente descritas, también se encuentran las fuentes sísmicas local y continental. De acuerdo con Rosenblueth et al. (1989), los eventos locales afectan sólo una pequeña zona, y los eventos que impactarían cualquier ubicación del Distrito Federal tienen periodos de retorno significativamente mayores a aquellos correspondientes a la región completa. Asimismo, los eventos continentales, están asociados con periodos de retorno superiores a los 1000 años. Como el peligro sísmico asociado con este tipo de eventos se considera bajo, éstos no son considerados en este estudio.

La distribución de probabilidad de la magnitud y la tasa de ocurrencia anual para cada una de las zonas mostradas en la figura 1 son presentadas en la tabla 1. Estos valores fueron tomados de Rosenblueth et al. (1989), Ordaz y Reyes (1999), y Jaimes y Reinoso (2006) (las coordenadas para las zonas que son inferidas de las dos últimas referencias están tabuladas en García-Soto et al. 2012).

La forma funcional de las relaciones de atenuación adoptadas en el presente estudio para sitios fuera del Valle de México y del Cinturón Volcánico Mexicano (CVM) (véase figura 1d) (Arroyo et al. 2010) se muestra en la tabla 2, donde $Y$ denota la aceleración espectral, $S_{A}\left(T_{\mathrm{n}}, \xi\right)$ en Gal; $T_{\mathrm{n}}$ (s) es el periodo fundamental de vibrar; $\xi$ es el porcentaje de amortiguamiento crítico, el cual es igual a $5 \%$ en este estudio. Las formas funcionales para logaritmo de $S_{A}\left(T_{\mathrm{n}}, \xi\right)$ correspondientes a sismos interplaca e intraslab son las sugeridas por García (2006) , y García et al. (2005), respectivamente.

Los coeficientes para las Ecuaciones (T2.a) y (T2.b), tomados de Hong et al. (2009b), se ilustran en la tabla 2 sólo para 3 periodos de vibrar, aunque los coeficientes para otros periodos de vibrar están disponibles en la referencia citada. Estos coeficientes fueron estimados usando el algoritmo propuesto por Joyner y Boore (1993) para la media geométrica. El uso de la media geométrica se justifica, ya que en este caso el término de error $\varepsilon$ representa la incertidumbre asociada en la predicción del movimiento del terreno para una orientación aleatoria (Boore et al. 1997). Nótese que en todos los casos, la desviación estándar de $\varepsilon, \sigma$, para las ecuaciones de regresión desarrolladas con base en la media geométrica (Hong et al. 2009b) es mayor que aquella basada en la media cuadrática dada por García et al. (2005) y García (2006).

Aunque relaciones de atenuación para la estación CU que consideran eventos interplaca están disponibles (Reyes et al. 2002, Pozos-Estrada et al. 2008), éstas no son consideradas debido a que no incluyen eventos más recientes. Sin embargo, la forma funcional de la relación de atenuación dada por Reyes et al. (2002), la cual es mostrada en la tabla 2, es adoptada. Para sismos intraslab, Montalvo-Arrieta (2002) propuso la forma funcional que es idéntica a la ecuación (T2.b), mostrada en la tabla 2, excepto que el término asociado a la profundidad focal (i.e., $c_{5}$ ) es considerado igual a cero. Es importante notar que al no tomarse en cuenta la profundidad focal, existen implicaciones al estimar aceleraciones espectrales para distancias pequeñas entre la falla y el sitio de interés. Más específicamente, debido a que parte de la zona sismogénica adoptada para sismos intraslab se ubica por debajo del Distrito Federal, al no considerarse la profundidad focal se sobrestima el peligro sísmico para tales eventos. Para evitar esta sobrestimación, en lugar de adoptar la ecuación recomendada por Montalvo-Arrieta (2002), la ecuación sugerida por García et al. (2005) (i.e., ecuación T2.b, ver tabla 2) fue considerada.

Para desarrollar los coeficientes de las relaciones de atenuación para la estación CU, se emplearon los eventos sísmicos de las tablas 3 y 4 correspondientes a sismos interplaca e intraslab, respectivamente. La lista de eventos mostrados en las tablas fue desarrollada con base en estudios de Reyes et al. (2002), Montalvo-Arrieta et al. (2002), y Singh et al. (2013). Debido a que hay varios registros disponibles de 
estaciones de registro cercanas entre ellas dentro de Ciudad Universitaria para los sismos ocurridos el 19 y 21 de septiembre de 1985, sólo un registro de la estación denotada como CU01 para cada uno de los eventos mencionados es usado. Para los registros considerados, un análisis de regresión empleando el algoritmo de Joyner y Boore (1993) (Hong et al. 2009a) se lleva a cabo considerando los eventos mostrados en la tabla 3, y sus correspondientes acelerogramas obtenidos para la estación CU.
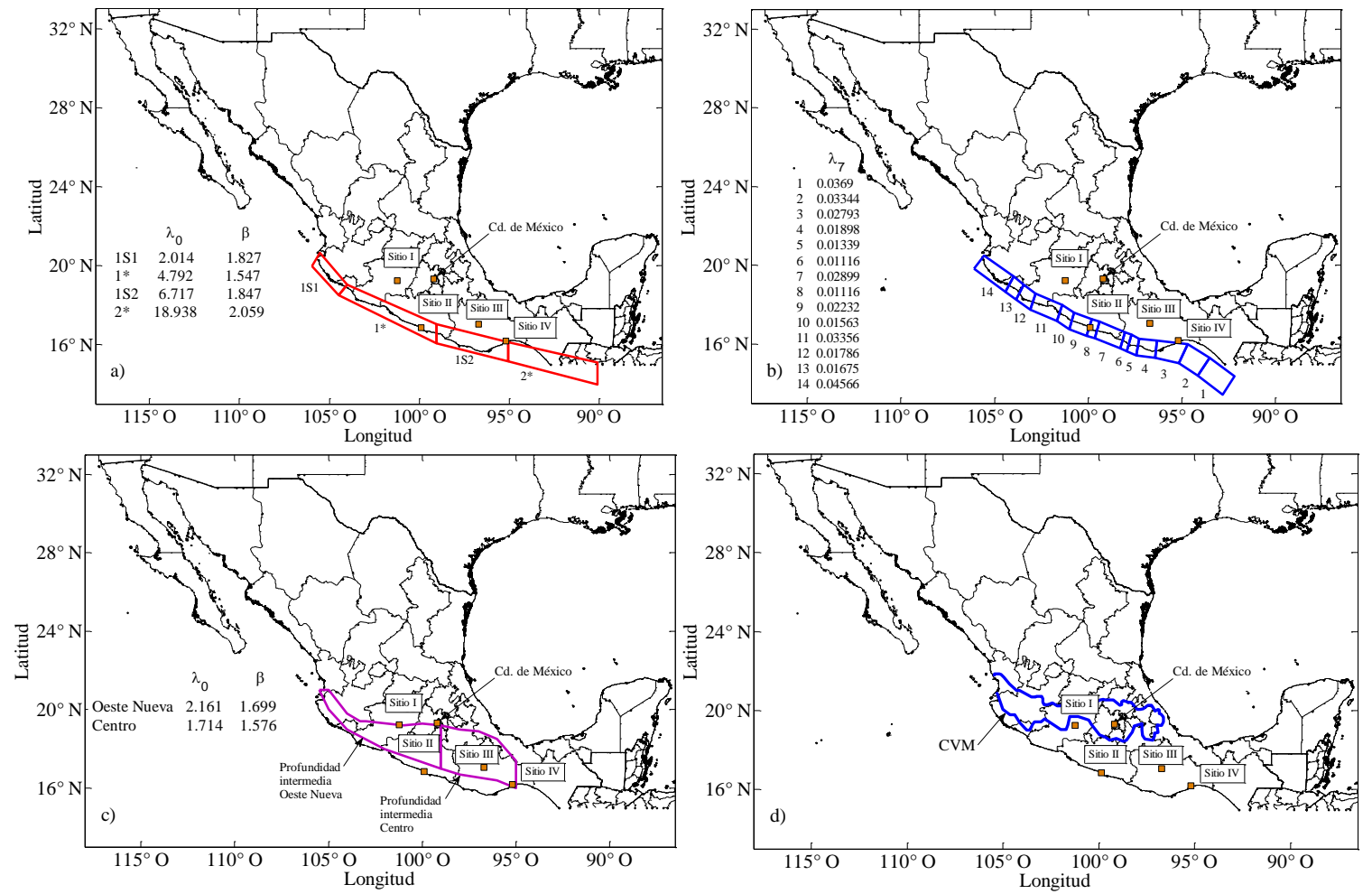

Figura 1. Zonas sismogénicas: a) sismos interplaca con $M_{\mathrm{W}} \leq 7$, b) sismos interplaca con $M_{\mathrm{W}}>7$, c) sismos intraslab, d) Cinturón Volcánico Mexicano (véase Arroyo et al. (2010))

Tabla 1. Relación magnitud-recurrencia para las zonas sismogénicas mostradas en la figura 1 (después de Ordaz y Reyes 1999, Jaimes y Reinoso 2006)

\begin{tabular}{ccc}
\hline Fuente sísmica & Relación magnitud-recurrencia & Intervalo de $M_{W}$ \\
\hline $\begin{array}{c}\text { Interplaca } \\
\text { (Fig. 2a) }\end{array}$ & $\lambda\left(M_{W}\right)=\lambda_{0} \frac{\exp \left(-\beta M_{W}\right)-\exp (-7 \beta)}{\exp (-4.5 \beta)-\exp (-7 \beta)}$ & $4.5-7.0$ \\
\hline $\begin{array}{c}\text { Interplaca } \\
\text { (Fig. 2b) }\end{array}$ & $\lambda\left(M_{W}\right)=\lambda_{7}\left(1-\Phi\left(\left(M_{W}-7.5\right) / 0.3\right)\right)$ & $M_{\mathrm{W}}>7$ \\
\hline $\begin{array}{c}\text { Intraslab: profundidad intermedia Oeste } \\
\text { (Fig. 2c) }\end{array}$ & $\lambda\left(M_{W}\right)=\lambda_{0} \frac{\exp \left(-\beta M_{W}\right)-\exp (-7.9 \beta)}{\exp (-4.5 \beta)-\exp (-7.9 \beta)}$ & $4.5-7.9$ \\
\hline $\begin{array}{c}\text { Intraslab: profundidad intermedia Centro } \\
\text { (Fig. 2c) }\end{array}$ & $\lambda\left(M_{W}\right)=\lambda_{0} \frac{\exp \left(-\beta M_{W}\right)-\exp (-7.88 \beta)}{\exp (-4.5 \beta)-\exp (-7.88 \beta)}$ & $4.5-7.88$ \\
\hline
\end{tabular}

Notas: El valor en la tabla 2 en Ordaz y Reyes (1999) es interpretado como $\lambda_{7} ; \lambda\left(M_{W}\right)$ representa la tasa de ocurrencia de un sismo con magnitud mayor o igual a $M_{W}$. Los parámetros $\lambda_{0}, \lambda_{7}$ y $\beta$ se muestran en la figura 1 .

Los coeficientes de la regresión considerando los sismos interplaca se presentan en la figura 2 así como en la tabla 2 para algunos valores de $T_{\mathrm{n}}$. Es interesante notar que los valores de $\sigma$ de la relación de atenuación para los acelerogramas obtenidos en la estación CU para los sismos interplaca son de alrededor 
del 60\% de aquellos asociados con la ecuación (T2.a). Esto es consistente con los reportados por PozoEstrada et al. (2008), y concuerda con la observación hecha por Atkinson (2006) que indica que para el mismo tipo de sismo $\sigma$ en una sola estación es de alrededor del $60 \%$ que aquel obtenido de los acelerogramas en una región.

Tabla 2. Coeficientes de relaciones de atenuación

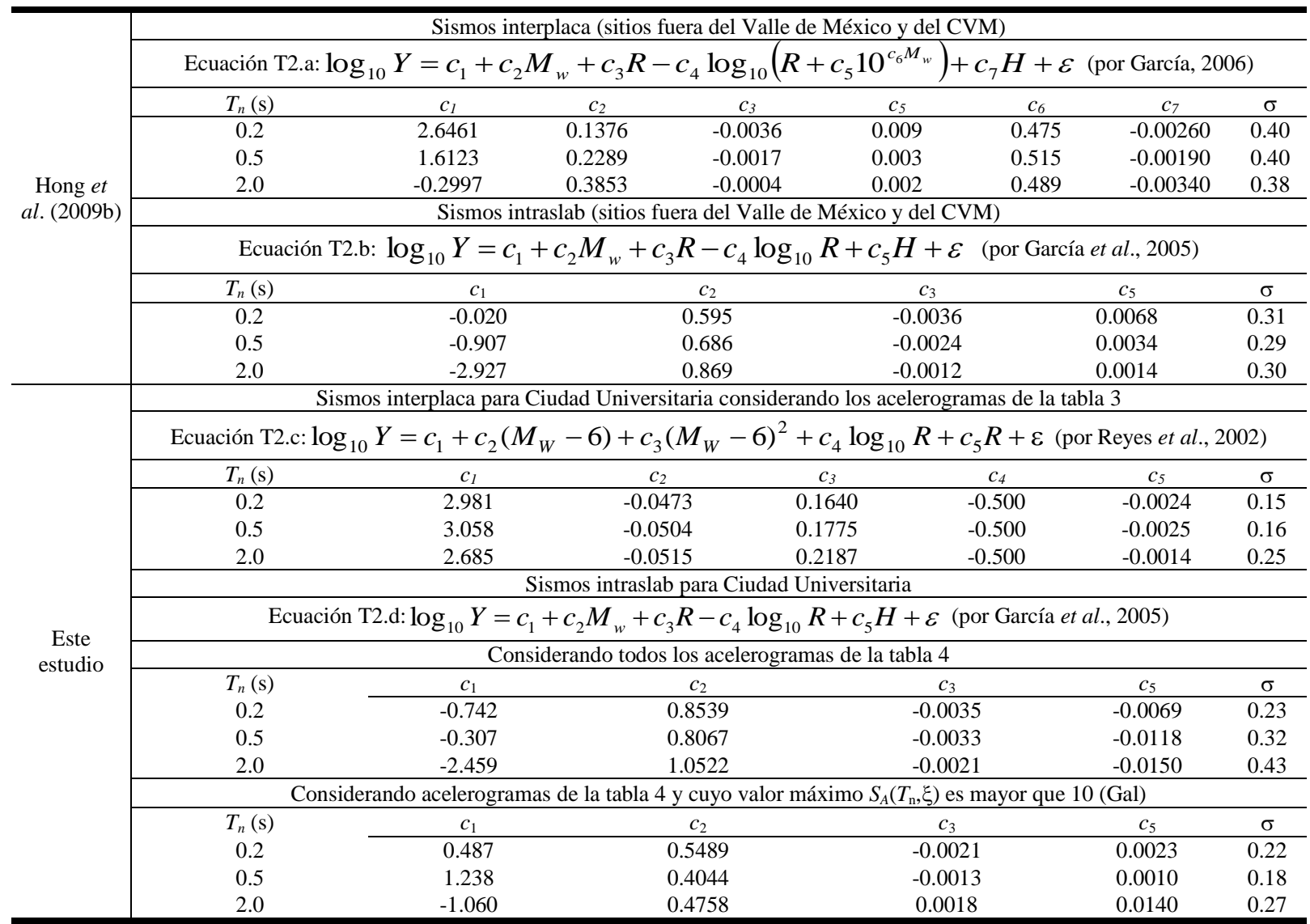

Notas para la tabla 2:

1) $\varepsilon$ en las ecuaciones es para una orientación aleatoria y es considerado como una variable normal con media cero y desviación estándar $\sigma$.

2) Para la relación de atenuación de sismos interplaca para sitios fuera del Valle de México y del Cinturón Volcánico Mexicano, $R$ (km) es la distancia más cercana a la superficie de falla para eventos con $M_{w}>6.0$, o la distancia hipocentral para el resto; $H$ (km) es la profundidad focal; $c_{4}=1.82-0.16 M_{w} ;$ y $c_{1}, c_{2}, c_{3}$, y $c_{7}$ son coeficientes de regresión. Se consideró un total de 413 acelerogramas con dos componentes horizontales obtenidos de 40 eventos sísmicos.

3) Para los sismos intraslab para sitios fuera del Valle de México y del Cinturón Volcánico Mexicano, $c_{\mathrm{i}}, i=1,2,3,4,5$, representa coeficientes de regresión; $c_{4}=1 ; R=\left(R_{\mathrm{cld}}{ }^{2}+\Delta_{0}\right)^{1 / 2} ; R_{\mathrm{cld}}(\mathrm{km})$ es la distancia más cercana a la superficie de falla para eventos con $M_{w}>6.5$, o la distancia hipocentral para el resto; $H(\mathrm{~km})$ es la profundidad focal; $\Delta_{0}=0.0075 \times 10^{0.507 \mathrm{M} w}$ es un término de saturación de la fuente definido por Atkinson y Boore (2003). Se consideraron 275 acelerogramas con dos componentes horizontales obtenidos de 16 eventos sísmicos.

4) Para la relación de atenuación para sismos interplaca para Ciudad Universitaria, $R(\mathrm{~km})$ es la distancia más cercana a la superficie de falla; $c_{\mathrm{i}}$, $i=1, \ldots, 5$ representan coefícientes de regresión. Se consideró un total de 18 acelerogramas con dos componentes horizontales obtenidos de 18 eventos sísmicos. La ecuación T2.c es válida para $M_{\mathrm{W}} \leq 8.1$; en caso de eventos con $M_{\mathrm{W}}>8.1$, sus magnitudes son consideradas igual a 8.1 (Reyes, comunicación personal 2007). La misma consideración es adoptada para la ecuación T2.a.

5) Para la relación de atenuación para sismos intraslab para Ciudad Universitaria ver 3) para las definiciones de los parámetros. Se consideraron 18 y12 acelerogramas con dos componentes horizontales obtenidos de 18 y 12 eventos sísmicos, respectivamente. La magnitud máxima para los sismos intraslab se considera menor o igual a 7.4, magnitud máxima observada en la base de datos empleada.

6) Se consideran profundidades deterministas de $22.22 \mathrm{~km}$ para sismos interplaca y $54.61 \mathrm{~km}$ para sismos intraslab. Estos valores representan la profundidad promedio de los sismos asociados con los registros utilizados en el desarrollo de las leyes de atenuación para la estación CU y son similares a los valores obtenidos con los registros utilizados para el desarrollo de las leyes de atenuación para sitios fuera del CVM y del Valle de México. Lo anterior se realiza ya que no se dispone de modelos probabilísticos asociados con esta variable. Nótese que en GarcíaSoto et al. (2012), el valor de la profundidad promedio para sismos interplaca, $10.45(\mathrm{~km})$, debería ser leído como $22.33(\mathrm{~km})$.

Para el caso de eventos intraslab, los espectros de los acelerogramas considerados se presentan en la 
figura 3. En la figura se incluyen los espectros para la media geométrica (figura 3 a y $3 b$ ) y para cada componente horizontal del terreno (figuras $3 \mathrm{c}, 3 \mathrm{~d}$, 3e y $3 \mathrm{f}$ ). Se observa de la figura 3 que los espectros presentan picos predominantes por debajo de 1 (s). En general, se observan picos asociados a periodos de vibrar cercanos a 0.2 y 0.5 (s). También se observa que los espectros de algunos sismos presentan un contenido importante de energía para periodos mayores a 1 (s). Lo anterior es consistente con lo observado por Montalvo-Arrieta (2002).

Tabla 3. Eventos sísmicos empleados en el desarrollo de la relación de atenuación para Ciudad Universitaria considerando sismos interplaca ( $h=$ profundidad $)$

\begin{tabular}{cccccccccc}
\hline Fecha & $M_{\mathrm{w}}$ & $h(\mathrm{~km})$ & Lat. N. & Long. O. & Fecha & $M_{\mathrm{w}}$ & $h(\mathrm{~km})$ & Lat. N. & Long. O. \\
\hline $23 / 08 / 1965$ & 7.8 & 33 & 15.38 & 96.12 & $19 / 09 / 1985$ & 8.1 & 15 & 18.08 & 102.94 \\
$03 / 02 / 1968$ & 5.6 & 33 & 16.37 & 99.40 & $21 / 09 / 1985$ & 7.6 & 15 & 18.02 & 101.48 \\
$02 / 08 / 1968$ & 7.4 & 33 & 16.07 & 98.10 & $30 / 04 / 1986$ & 7.0 & 20 & 18.02 & 103.06 \\
$19 / 03 / 1978$ & 6.4 & 36 & 17.03 & 99.76 & $25 / 04 / 1989$ & 6.9 & 19 & 16.60 & 99.40 \\
$29 / 11 / 1978$ & 7.8 & 19 & 16.00 & 96.69 & $31 / 05 / 1990$ & 6.1 & 16 & 17.11 & 100.89 \\
$14 / 03 / 1979$ & 7.6 & 28 & 17.49 & 101.26 & $24 / 10 / 1993$ & 6.7 & 19 & 16.54 & 98.98 \\
$25 / 10 / 1981$ & 7.3 & 14 & 17.88 & 102.15 & $10 / 12 / 1994$ & 6.6 & 20 & 18.02 & 101.56 \\
$07 / 06 / 1982$ & 6.9 & 18 & 16.17 & 98.36 & $14 / 09 / 1995$ & 7.3 & 22 & 16.31 & 98.88 \\
$07 / 06 / 1982$ & 7.0 & 24 & 16.26 & 98.51 & $20 / 03 / 2012$ & 7.4 & 16 & 16.25 & 98.52 \\
\hline
\end{tabular}

Tabla 4. Eventos sísmicos empleados en el desarrollo de la relación de atenuación para Ciudad Universitaria considerando sismos intraslab ( $h=$ profundidad)

\begin{tabular}{cccccccccc}
\hline Fecha & $M_{\mathrm{w}}$ & $h(\mathrm{~km})$ & Lat. N. & Long. O. & Fecha & $M_{\mathrm{w}}$ & $h(\mathrm{~km})$ & Lat. N. & Long. O. \\
\hline $06 / 07 / 1964$ & 6.7 & 55 & 18.03 & 100.77 & $03 / 04 / 1997$ & 5.2 & 52 & 18.51 & 98.10 \\
$07 / 06 / 1976$ & 6.4 & 57 & 17.09 & 100.85 & $22 / 05 / 1997$ & 6.5 & 54 & 18.37 & 101.82 \\
$24 / 10 / 1980$ & 7.0 & 70 & 18.03 & 98.29 & $20 / 04 / 1998$ & 5.9 & 60 & 18.35 & 101.19 \\
$05 / 08 / 1993$ & 5.2 & 54 & 17.43 & 98.337 & $15 / 06 / 1999$ & 7.0 & 61 & 18.13 & 97.54 \\
$23 / 02 / 1994$ & 5.8 & 75 & 17.75 & 97.27 & $21 / 06 / 1999$ & 6.3 & 53 & 18.15 \\
$06 / 05 / 1994$ & 5.2 & 57 & 18.39 & 97.98 & $30 / 09 / 1999$ & 7.4 & 47 & 16.03 \\
$23 / 05 / 1994$ & 6.2 & 50 & 18.02 & 100.57 & $21 / 07 / 2000$ & 5.9 & 50 & 18.11 & 96.96 \\
$10 / 12 / 1994$ & 6.4 & 50 & 17.98 & 101.52 & $22 / 05 / 2009$ & 5.7 & 45 & 18.13 \\
$11 / 01 / 1997$ & 7.1 & 35 & 18.37 & 101.82 & $11 / 12 / 2011$ & 6.5 & 58 & 17.84 & 98.44 \\
\hline
\end{tabular}
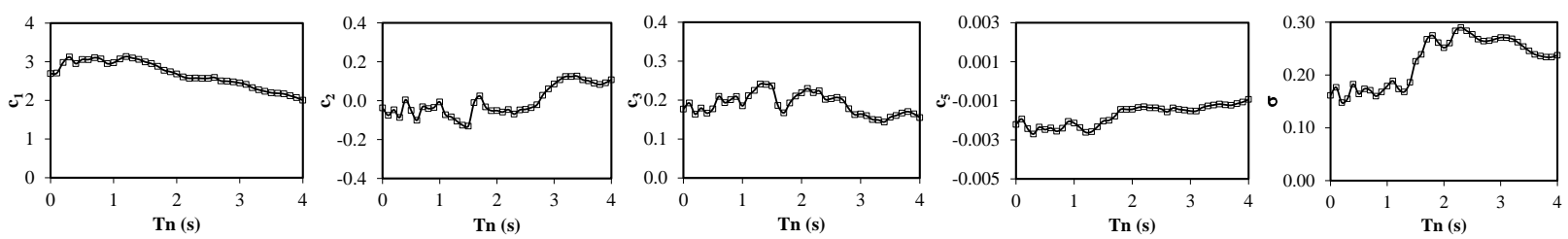

Figura 2. Coeficientes de regresión para sismos interplaca para la estación CU

Los resultados del análisis de regresión indican que los coeficientes de la regresión varían significativamente con el periodo natural de vibrar $T_{\mathrm{n}}$ como se muestra en la figura 4 (ver también la tabla 2). También se muestra en la figura 4 la desviación estándar $\sigma$. La figura indica que $\sigma$ aumenta conforme $T_{\mathrm{n}}$ aumenta, sus valores aumentan hasta 0.5 lo cual es significativamente mayor que aquellos para la ecuación (T2.b) que fueron desarrollados con base en eventos intraslab, pero para registros de muchas estaciones. Esto es inesperado ya que $\sigma$ para una sola estación considerando el mismo tipo de sismo debe ser menor que aquel asociado con muchas estaciones (Atkinson 2006). Una inspección de los residuos $\varepsilon$ del análisis de regresión indica que éstos se desvían significativamente de una variable normal, lo que indica que el modelo de regresión es inadecuado para los registros considerados.

Para mejorar el ajuste usando el mismo modelo adoptado, los registros cuyo valor máximo de $S_{A}\left(T_{\mathrm{n}}, \xi\right)$, incluyendo la PGA, es menor que 10 (Gal) fueron eliminados. Esto resultó en sólo 12 registros para la estación CU. En este caso, los coeficientes y $\sigma$ obtenidos se muestran en la figura 5 así como en la 
tabla 2 para algunos valores seleccionados de $T_{\mathrm{n}}$. La comparación de los resultados mostrados en las figuras 4 y 5 indica que al eliminar los registros cuyo valor máximo de $S_{A}\left(T_{\mathrm{n}}, \xi\right)$ es menor que 10 (Gal), los valores de $\sigma$ son menores a los obtenidos considerado todos los registros. También se observa que el valor máximo de $\sigma$ para el caso seleccionado es aproximadamente igual a 0.30. Este valor es comparable con aquellos obtenidos fuera del Valle de México y del CVM basados en una amplia red de estaciones para sismos intraslab como se muestra en la tabla 2.

Aunque se puede desarrollar una relación de atenuación con base en los registros con excitaciones mayores que un límite inferior mayor que $10(\mathrm{Gal})$, esto no fue considerado ya que se reduciría el número de acelerogramas que pueden ser usados para desarrollar las relaciones de atenuación. La consideración de un límite inferior de 10 (Gal) en el desarrollo de la relación de atenuación puede ser una suposición conservadora. Debido a que la selección de un límite inferior de 10 (Gal) es de alguna forma arbitraria, con fines de comparación, la evaluación del peligro sísmico para la estación CU es llevada a cabo en las siguientes secciones para los casos CU-1 y CU-2. El caso CU-1 se refiere a la evaluación del peligro sísmico empleando la relación de atenuación desarrollada para registros de eventos intraslab con valores máximos de $S_{A}\left(T_{\mathrm{n}}, \xi\right)$ mayores que $10(\mathrm{Gal})$, mientras que el caso CU-2 se refiere a la evaluación del peligro sísmico considerando la relación de atenuación desarrollada con base en todos los eventos intraslab mostrados en la tabla 4.
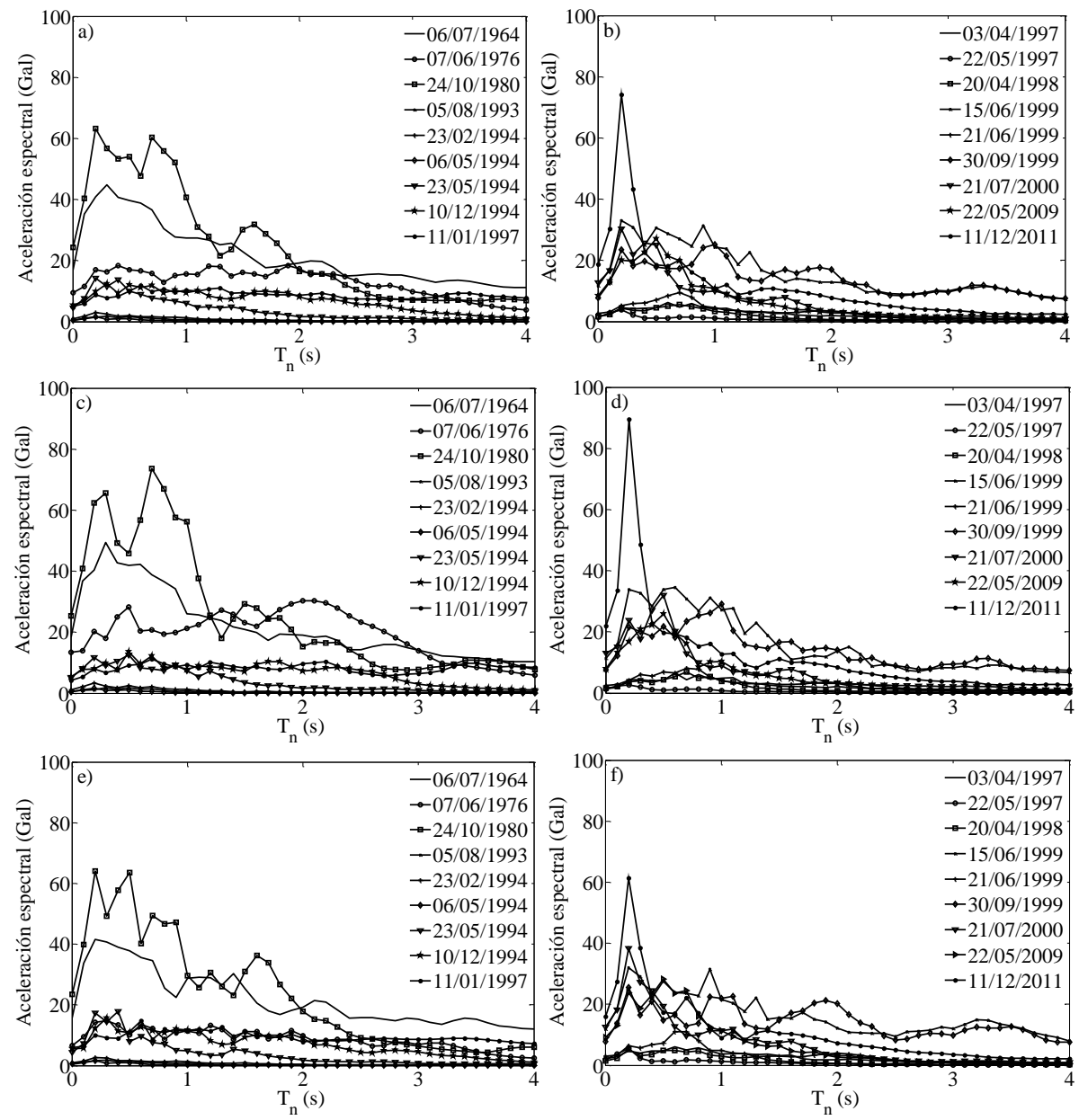

Figura 3. Espectros de respuesta para sismos intraslab para la estación CU ( $\xi=5 \%$ ): a) y b) para la media geométrica; c) y d) primer componente horizontal; e) y f) segunda componente horizontal 

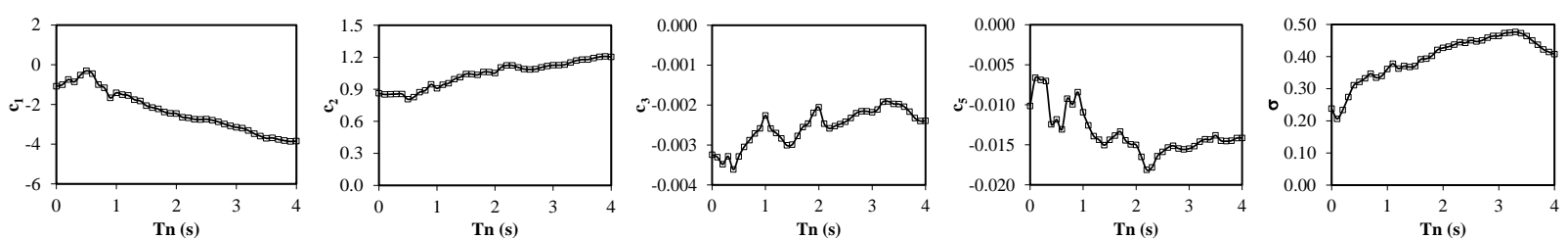

Figura 4. Coeficientes de regresión para sismos intraslab para la estación CU, considerando todos los acelerogramas
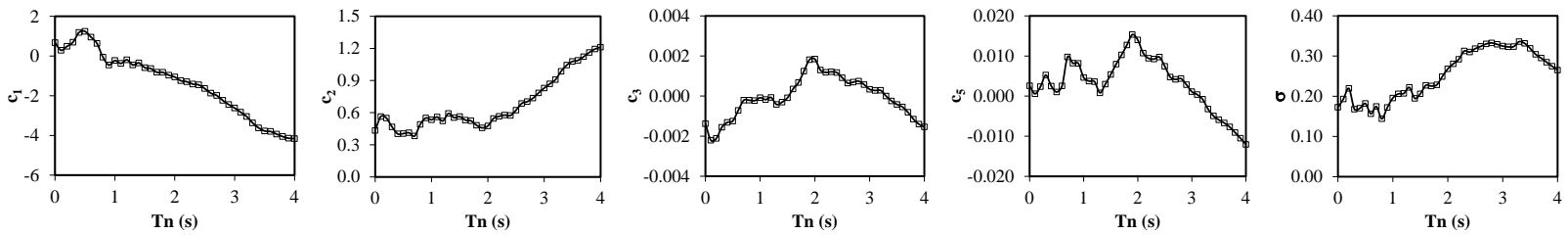

Figura 5. Coeficientes de regresión para sismos intraslab para la estación CU, considerando acelerogramas cuyo valor máximo de $S_{A}\left(T_{\mathrm{n}}, \xi\right)$ es mayor que $10(\mathrm{Gal})$

\section{MAPAS DE CONTORNO, ESPECTROS DE PELIGRO UNIFORME Y RESULTADOS DE LA DESAGREGACIÓN}

\section{Procedimiento para estimar y desagregar el peligro sísmico}

Una vez que un modelo de peligro sísmico es asignado, el peligro sísmico en el sitio de interés, definido por la distribución de probabilidad de las mediciones del movimiento del terreno, puede ser evaluado, y el análisis de desagregación se puede llevar a cabo. Uno de los procedimientos más populares para la estimación probabilista del peligro sísmico (EPPS) fue desarrollado por Esteva (1968) y Cornell (1968). Este procedimiento se ilustra en la figura 6. El algoritmo para evaluar el peligro sísmico en un sitio puede ejecutarse con base en integración numérica (Cornell 1968; McGuire 2004) o técnicas de Monte Carlo (Musson 2000; Hong et al. 2006). El uso de técnicas de Monte Carlo ofrece la ventaja de que éste puede ser empleado directamente para tratar con modelos de ocurrencia Poisonianos o no Poisonianos homogéneos o no homogéneos, y puede ser fácilmente adaptado para estimar espectros de peligro uniforme (EPU) y el análisis de desagregación de peligro o riesgo sísmico (Hong et al. 2006; Hong y Goda 2006).

La estimación del EPU consiste en encontrar las respuestas espectrales para diferentes periodos naturales de vibrar con una probabilidad de excedencia específica (i.e., fractiles de $S_{A}\left(T_{n}, \xi\right)$ denotados por $\left.\mathrm{S}_{A f}\left(T_{n}, \xi\right)\right)$. La desagregación del peligro sísmico consiste en identificar la combinación de valores de las variables aleatorias que caracterizan a los eventos sísmicos que contribuyen a $\mathrm{S}_{A f}\left(T_{n}, \xi\right)$. A menudo, las variables consideradas para la desagregación son la magnitud, la distancia de la fuente al sitio de interés, y $\varepsilon$ en la relación de atenuación. Además de estas tres variables, también incluimos el tipo de sismo y la profundidad del sismo en el análisis de desagregación. Los pasos para el análisis son los siguientes:

1) Llevar a cabo la simulación para determinar muestras de $S_{A}\left(T_{n}, \xi\right)$ para un periodo de vibrar y amortiguamiento dados;

2) Encontrar $S_{A f}\left(T_{n}, \xi\right)$ mediante un análisis probabilístico de las muestras; 
3) Recolectar todos los eventos cuya respuesta sea igual o cercana al valor de $S_{A f}\left(T_{n}, \xi\right)$, y llevar a cabo un análisis estadístico con los parámetros que caracterizan a los eventos recolectados.

Los primeros dos pasos para el análisis son iguales a los mostrados en la figura 6 , y son empleados en los análisis numéricos.

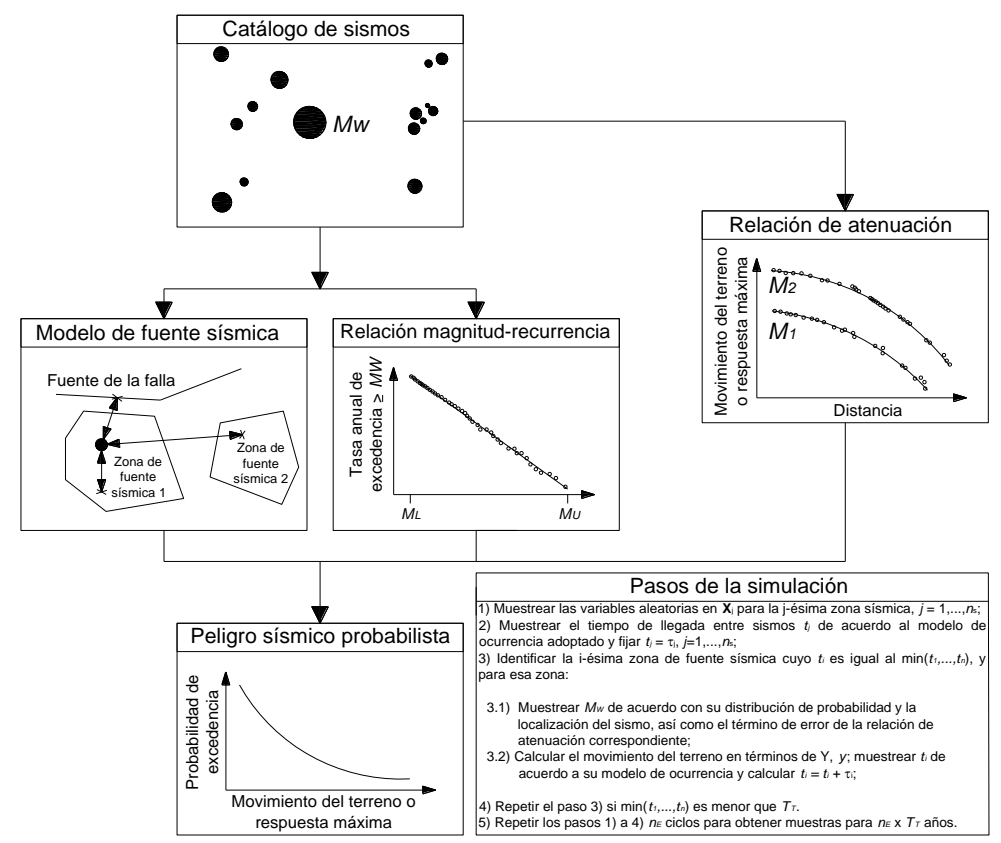

Figura 6. Ilustración del método del cálculo del peligro sísmico (Modificado de García-Soto et al. 2012, y Hong 2012)

\section{Mapas de contorno y espectros de peligro uniforme}

Se elaboraron mapas de contorno de $S_{A f}\left(T_{n}, \xi\right)$ para evaluar el peligro sísmico en algunas regiones de México, incluyendo al Distrito Federal en terreno firme (Ciudad Universitaria). Para el cálculo del peligro símico se empleó el método de simulación descrito en secciones anteriores. Los valores simulados representan 187,500 años de actividad sísmica. Los resultados de $S_{A f}\left(T_{\mathrm{n}}, \xi\right)$ para un periodo de retorno igual a 125 años, considerando la opción CU-1 para la estación CU, se presentan en la figura 7. Para evitar confusión en la lectura de los niveles de aceleración presentados en las gráficas de la figura 7, éstas deben asociarse con la graduación de aceleración correspondiente, cuyo valor máximo difiere para cada caso. La figura 7 muestra el CVM sombreado en blanco desvanecido (ver figura 2d). Es importante recalcar que los resultados de la simulación del peligro sísmico, determinados con las relaciones en la tabla 2, no deben ser extrapolados al CVM ni al Valle de México (excepto para la estación CU), debido a las características particulares de propagación a lo largo de estas regiones (García 2006). Se observa de la figura 7 que, como se esperaba, el peligro sísmico es mayor para sitios cercanos a la costa. Se observa también que el impacto del peligro sísmico es mayor para estructuras con $T_{n}=0.2$ (s) y que éste disminuye para estructuras con $T_{n}$ igual a 0.5 y $2(\mathrm{~s})$, en particular para $T_{n}=2(\mathrm{~s})$, se observa una reducción significativa del peligro sísmico.

Aunque mapas para periodos de retorno iguales a 475, 1000 y 2475 años también fueron evaluados para diferentes valores de $T_{\mathrm{n}}$, estos no son presentados ya que son idénticos (excepto para la estación en Ciudad Universitaria) a los presentados por García-Soto et al. (2012). 
Para apreciar mejor el peligro sísmico para diferentes periodos naturales de vibrar, de forma similar a García-Soto et al. (2012), calculamos el EPU para la estación CU considerando un periodo de retorno igual a 125 años y dos opciones para los sismos de intraslab (CU-1 y CU-2). También calculamos el EPU para otros cuatro lugares que se localizan cerca de Morelia, Acapulco, Oaxaca y Salina Cruz (véase la figura 1). Estos lugares se refieren como el Sitio I, II, III y IV en lo siguiente. Los resultados obtenidos son mostrados en la figura 8 . Se observa de la figura que, al igual que en los mapas de contorno, el peligro sísmico es más significativo para los sitios más cercanos a la costa y para $T_{n}$ cercano a 0.2 (s). También se observa que la forma de los EPU para los Sitios I a IV es similar. El EPU para la estación CU mostrado en la figura 8 es debido a sismos intraslab e interplaca. Se observa que este EPU tiene una forma muy diferente que los EPU para los Sitios I a IV, principalmente para $T_{n}$ menores a 1.7 (s), en donde se aprecian periodos de vibrar característicos con un peligro sísmico importante para el valle de México. El EPU para el Sitio CU, usando CU-1, es comparable en magnitud con los EPU para los Sitios I a IV para $T_{n}$ grandes. Asimismo, la figura muestra que hay diferencias marcadas entre el EPU obtenido usando la opción CU-1 y CU-2. El EPU empleando CU-2 es mucho más grande que aquel obtenido con CU-1; esto indica que el peligro sísmico en la estación CU es altamente afectado por la relación de atenuación adoptada, incluyendo $\varepsilon$, para sismos intraslab.
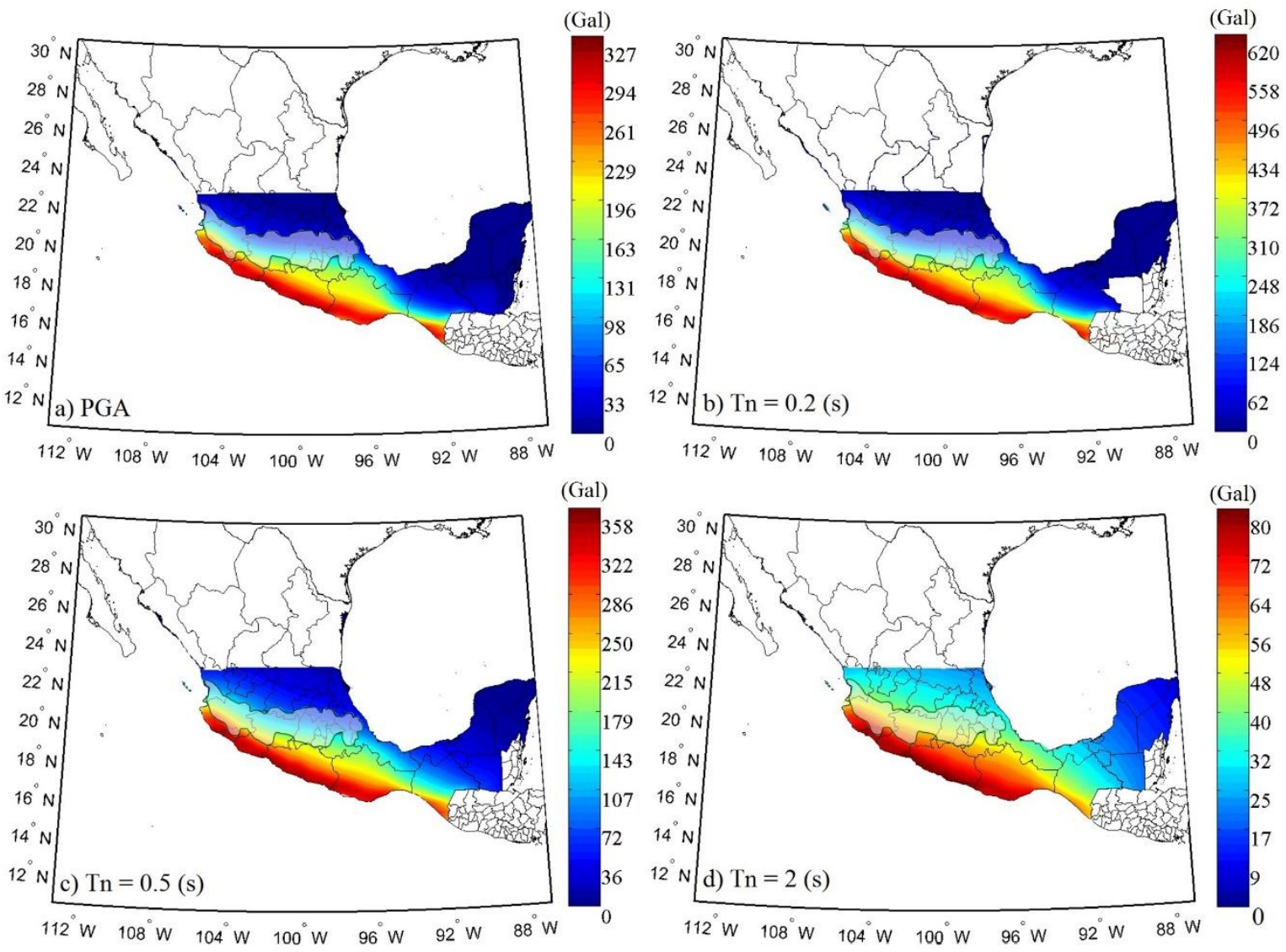

Figura 7. Mapas de contorno considerando sismos interplaca e intraslab para un periodo de retorno igual a 125 años

Para apreciar mejor la influencia de la relación de atenuación y los sismos intraslab e interplaca en el peligro sísmico, calculamos nuevamente el EPU debido sólo a sismos interplaca, sólo a sismos intraslab (con CU-1 o CU-2) y los sismos interplaca e intraslab para periodos de retorno iguales a 125 y 475 años. 
Los valores obtenidos son mostrados en la figura 9 y comparados con el espectro de diseño recomendado por las Normas Técnicas Complementarias para el Diseño por Sismo (NTCDS) (Diario Oficial de la Federación, 2004) para la Ciudad de México para terreno firme. Las gráficas mostradas en la figuras 9a y $9 \mathrm{~b}$ indican que los sismos interplaca e intraslab contribuyen al peligro sísmico en la estación CU. Asimismo, el uso de CU-2 para sismos intraslab sobrestima el peligro sísmico comparado con aquellos usando CU-1. Esto claramente indica la importancia de usar una relación de atenuación (y $\varepsilon$ ) apropiada para la evaluación del peligro sísmico; el uso de una relación de atenuación tal como la CU-2 es cuestionable y puede llevar a obtener un peligro sísmico erróneo. Por lo tanto, el uso de CU-2 no es considerado más en lo siguiente.

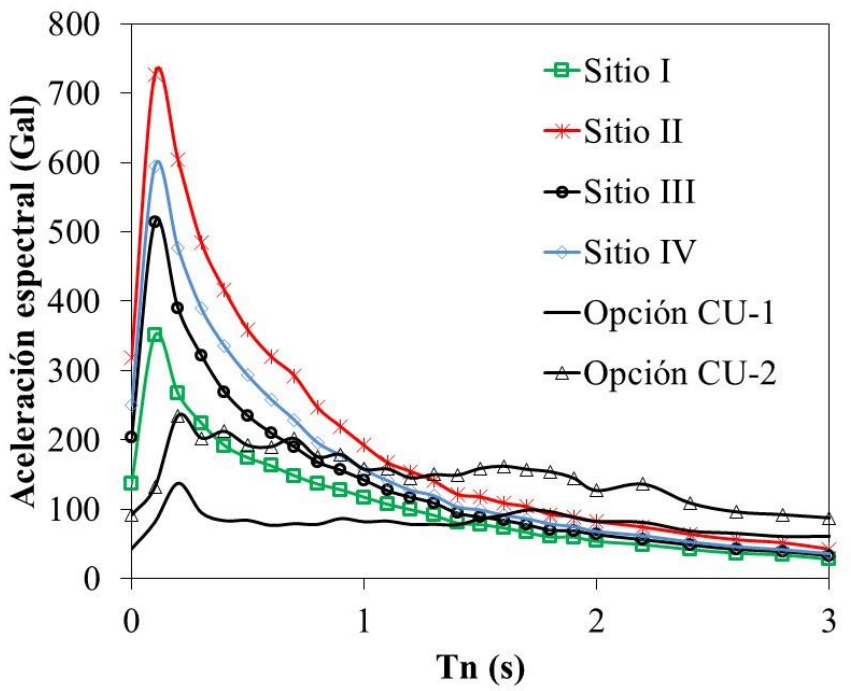

Figura 8. Espectros de peligro uniforme para los Sitios I, II, III, IV y para la estación CU (empleando las opciones CU-1 y CU-2) para un periodo de retorno de 125 años
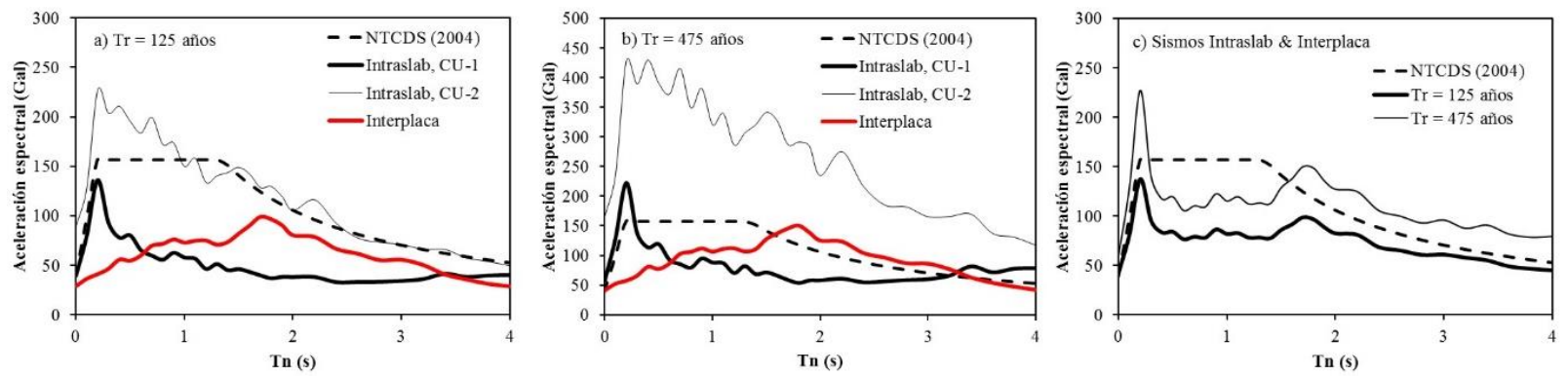

Figura 9. Comparación de espectros de peligro uniforme con el espectro de diseño para terreno firme de las Normas Técnicas Complementarias

Nótese que al considerar CU-1 para eventos intraslab, se observa en la figura 9a que las ordenadas espectrales del EPU para sismos intraslab son similares a las del espectro de diseño hasta un periodo de vibrar cercano a 0.2 (s), después de este periodo las ordenadas espectrales disminuyen hasta $T_{n}$ cercano a 1 (s), donde se observa nuevamente un leve incremento en las ordenadas espectrales, después de este periodo de vibrar las ordenadas espectrales del EPU disminuyen gradualmente para periodos grandes. Se observa en la misma figura que el EPU para sismos interplaca presenta ordenadas espectrales máximas para periodos de vibrar cercanos a 1 y a 1.7 (s). Los EPU para sismos intraslab e interplaca se cruzan en $T_{n}=0.7$ (s), aproximadamente, indicando que el peligro sísmico para una estructura con un periodo 
fundamental menor que 0.7 (s) es dominado por sismos intraslab mientras el peligro sísmico para estructuras con un periodo fundamental mayor que 0.7 (s) es dominado por sismos interplaca. Las ordenadas espectrales de los EPU de sismos intraslab o de sismos interplaca son menores a las del espectro de diseño para el periodo de retorno de 125 años.

Similar a la figura 9a, la figura 9b presenta la comparación de los EPU pero para un periodo de retorno de 475 años. En general, las tendencias observadas en figura 9a son igualmente aplicables a figura $9 \mathrm{~b}$, excepto que, y como era de esperarse, las ordenadas del EPU en la figura $9 \mathrm{~b}$ son mayores que aquellas de la figura 9 a.

La figura 9c muestra los EPU considerando la contribución de los sismos intraslab e interplaca para periodos de retorno iguales a 125 y 475 años, empleando sólo CU-1 para sismos intraslab. La figura indica que el espectro de diseño para terreno firme de NTCDS (Diario Oficial de la Federación, 2004) es mayor que el EPU para a un periodo de retorno de 125 años y, el espectro de diseño es más grande que el EPU para un periodo de retorno de 475 años para $T_{n}$ entre 0.25 y 1.75 (s). Esta observación difiere de la conclusión que el espectro de diseño corresponde a un EPU para un periodo de retorno de 125 años (Ordaz et al. 2000). La diferencia podía ser explicada al notar que registros adicionales de eventos sísmicos más recientes son considerados en el presente estudio.

\section{Resultados de la desagregación}

Como se mencionó previamente, la desagregación sísmica consiste en identificar combinaciones de variables aleatorias que definen eventos sísmicos que contribuyen a un nivel de peligro sísmico predefinido. Estas variables aleatorias incluyen $M_{w}, R$ y $\varepsilon$, empleados en las relaciones de atenuación.

La desagregación del peligro sísmico se realiza para los sitios identificados como I, II, III y IV, junto con la estación CU, para la aceleración máxima del terreno y la aceleración espectral para $T_{n}=0.2$, 0.5 , y 2 (s). Los resultados obtenidos de la desagregación del peligro sísmico para los sitios considerados se presentan en las figuras 10 a 14 para periodos de retorno iguales a 125 y 475 años. En las figuras se presenta la contribución de cada tipo de sismo (intraslab o interplaca) al peligro sísmico del sitio en estudio. Las gráficas mostradas en las figuras consideran un intervalo de 0.5 para $M_{w}$ y de $25(\mathrm{~km})$ para $R$. También en la figuras se identifican escenarios sísmicos con valores modales de $\left(M_{w}, R, \varepsilon\right)$.

La figura 10 muestra la desagregación sísmica para la estación CU, en donde se observa que la función de masa de probabilidad parece ser unimodal. Los parámetros modales que identifican a los escenarios sísmicos son $(7.4,150,0.18),(7.3,147,0.23),(7.3,149,0.21),(7.8,296,0.34)$ para la aceleración máxima del terreno y las aceleraciones espectrales para $T_{n}=0.2,0.5$, y 2 (s), respectivamente. Estos parámetros están asociados con un periodo de retorno de 125 años. Una inspección más detallada indica que la mayoría de los escenarios sísmicos asociados con la aceleración máxima del terreno y las aceleraciones espectrales para $T_{n}=0.2$ y 0.5 (s) provienen de las zonas sísmicas de eventos intraslab, aunque también se observa contribución de sismos interplaca para la aceleración máxima del terreno y la aceleración espectral para $T_{n}=0.5(\mathrm{~s})$. El escenario sísmico asociado con $T_{n}=2$ (s) tiene su contribución más importante de eventos interplaca, más particularmente de las zonas sísmicas de Guerrero (ver figura 1b). Los escenarios símicos identificados para un periodo de retorno de 475 años son diferentes pero no significativamente a aquellos para un periodo de retorno de 125 años.

En general, para los sitios I a IV (ver figuras 11 a 14), se observa contribución de eventos interplaca e intraslab al peligro sísmico, la contribución más importante de eventos interplaca es para los Sitios II y IV (cerca de Acapulco y Salina Cruz, respectivamente), mientras que los eventos interplaca e intraslab dominan el peligro sísmico para los Sitios I y III (cerca de Morelia y Oaxaca). Los escenarios sísmicos 
con parámetros modales identificados en la desagregación muestran que en algunos casos la contribución al peligro sísmico es dominada por un solo evento y en otros el peligro sísmico es debido a diferentes escenarios sísmicos y diferentes tipos de temblores. Esto es importante ya que la demanda de ductilidad podría diferir para diferentes tipos de sismos (Hong et al. 2010).
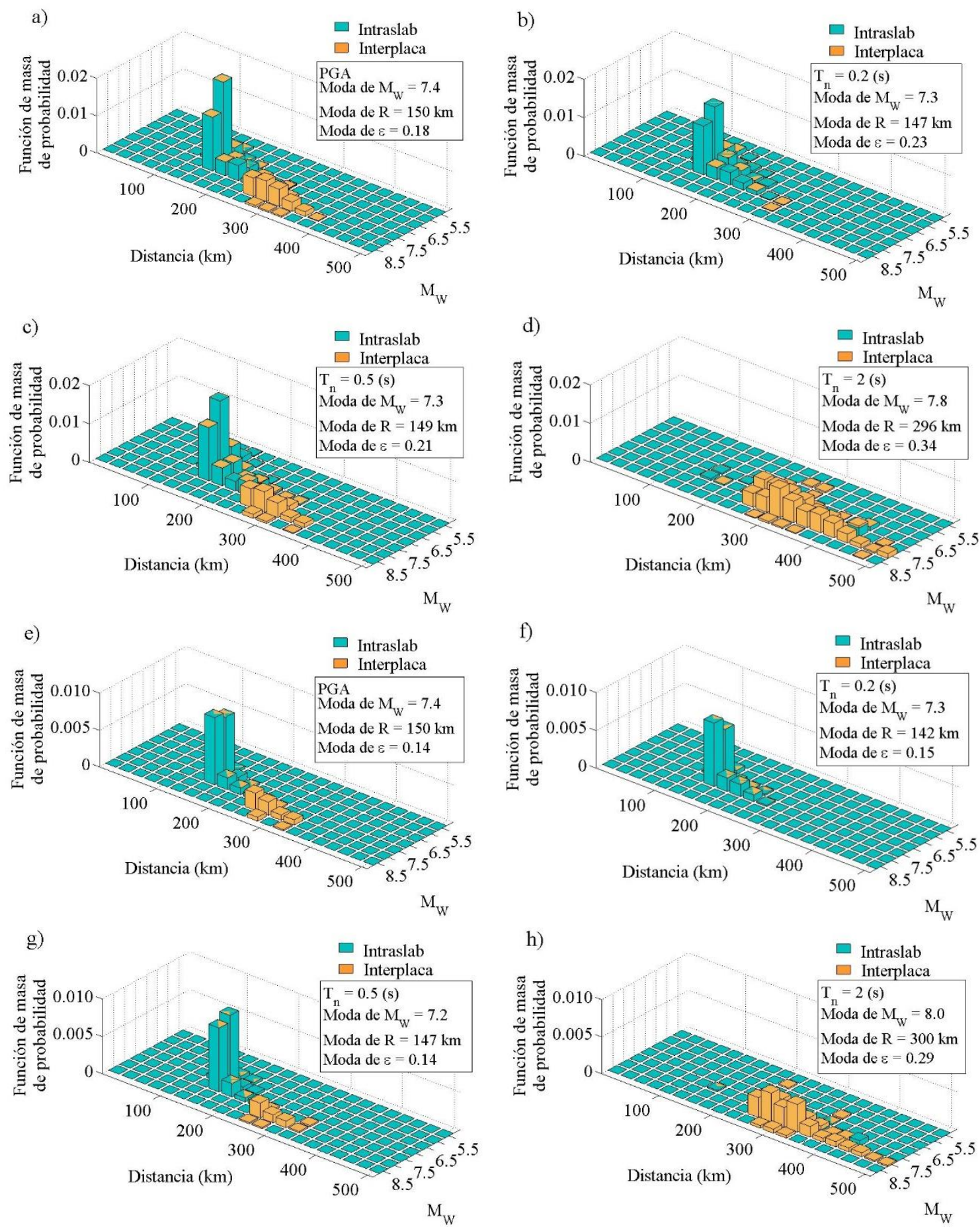

Figura 10. Desagregación sísmica para la estación CU para: (a) a (d) periodo de retorno de 125 años; (e) a (h) periodo de retorno de 475 años 

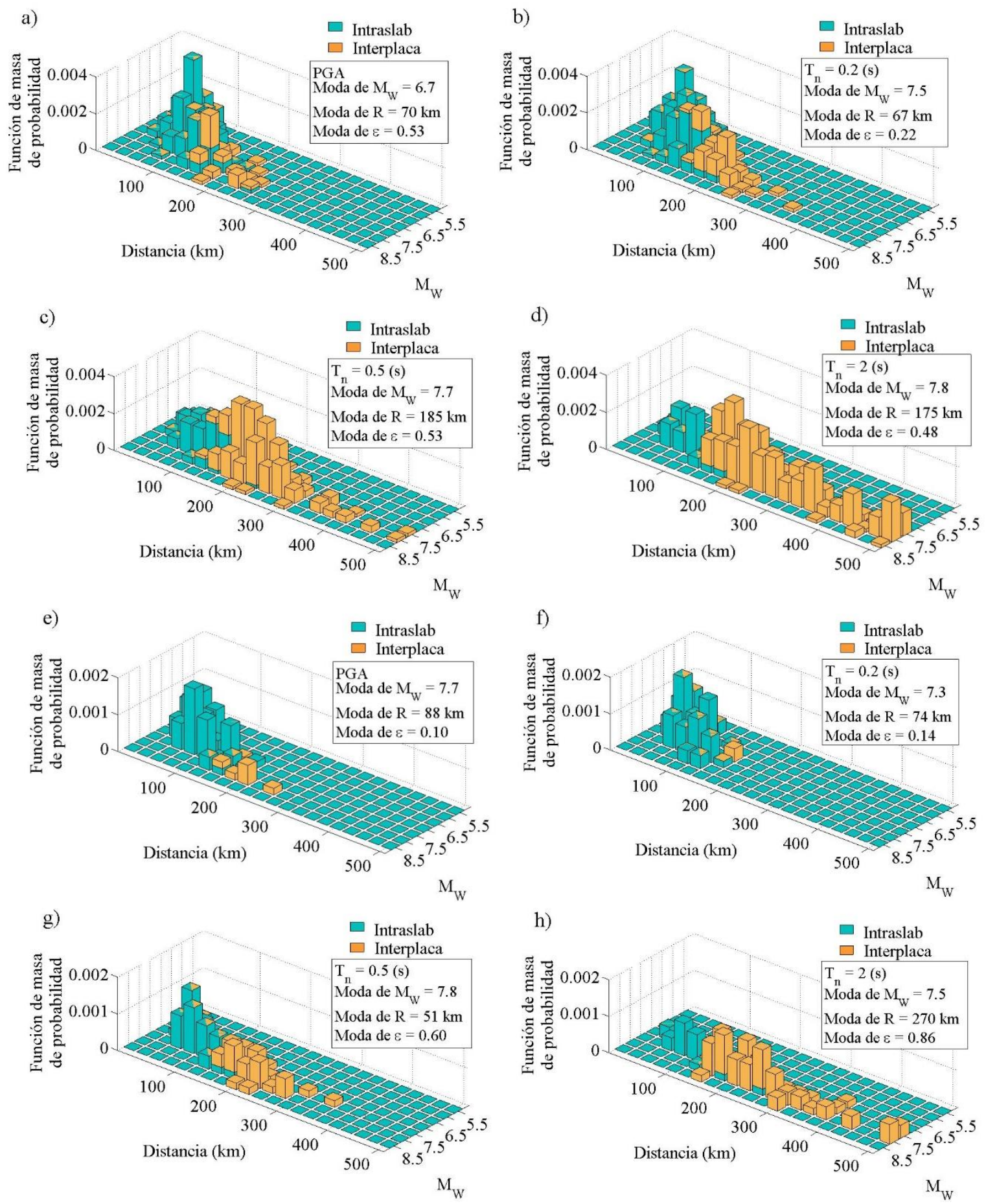

Figura 11. Desagregación sísmica para el Sitio I (cerca de Morelia): (a) a (d) periodo de retorno de 125 años; (e) a (h) periodo de retorno de 475 años 

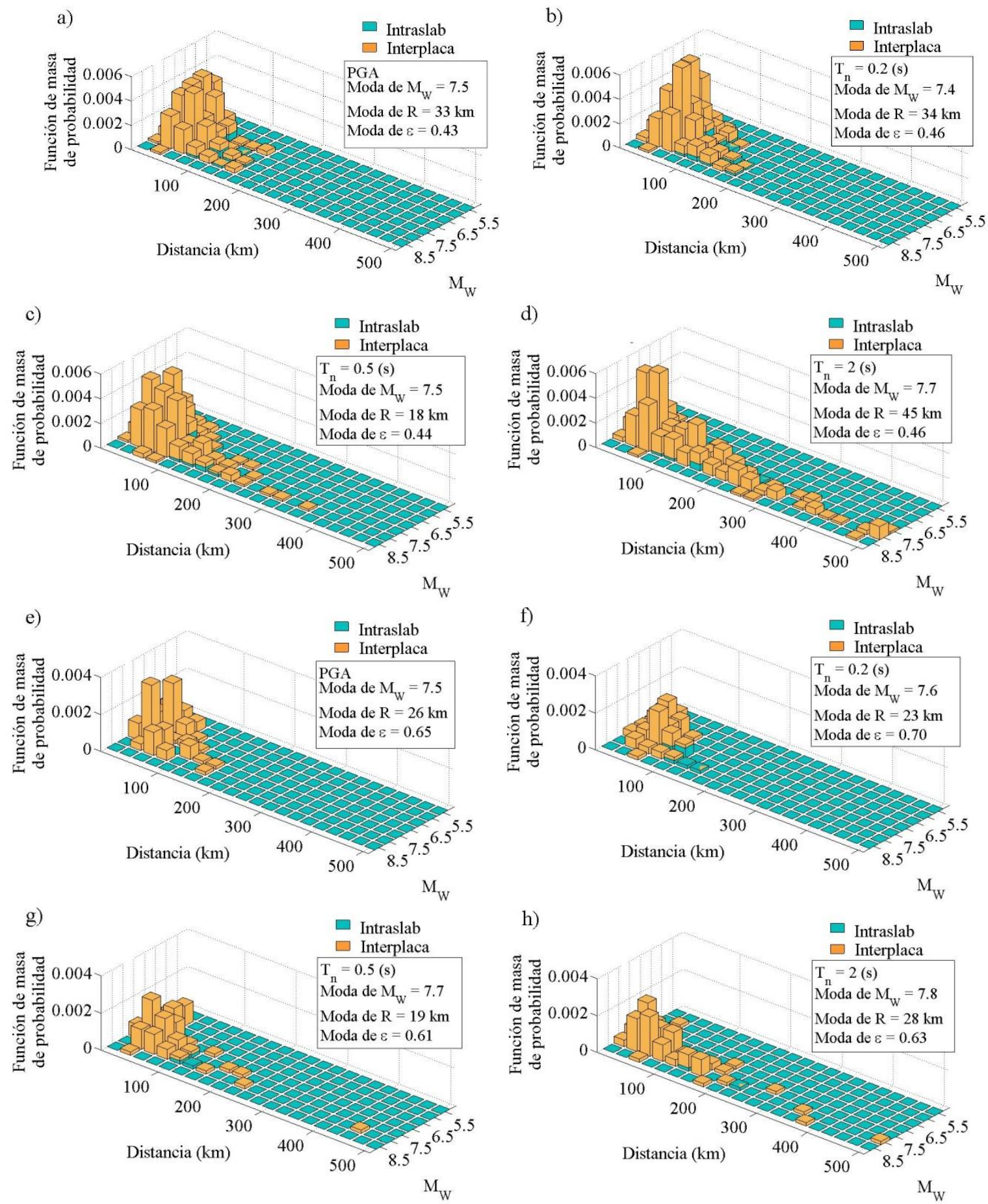

Figura 12. Desagregación sísmica para el Sitio II (cerca de Acapulco): (a) a (d) periodo de retorno de 125 años; (e) a (h) periodo de retorno de 475 años 

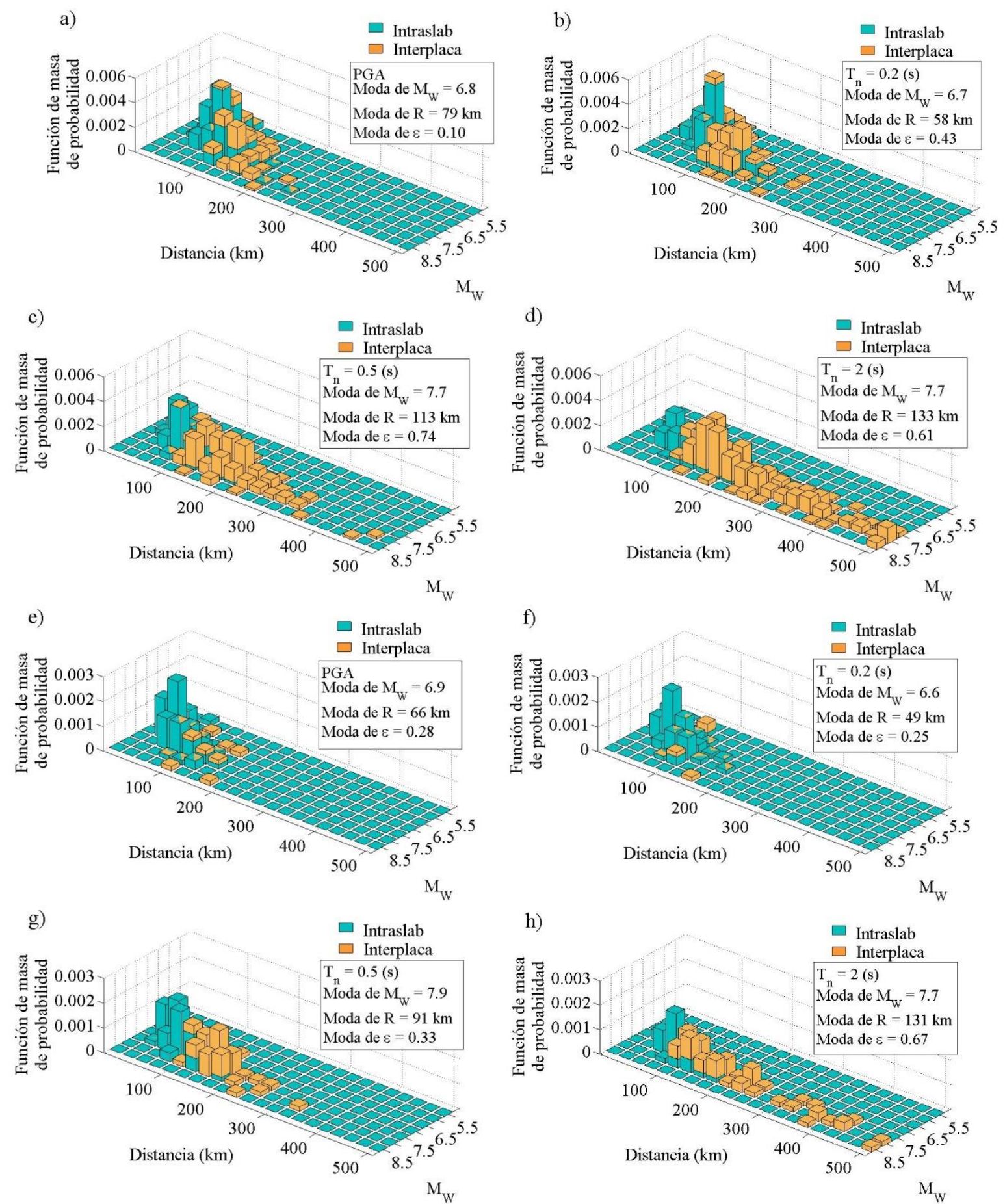

Figura 13. Desagregación sísmica para el Sitio III (cerca de Oaxaca): (a) a (d) periodo de retorno de 125 años; (e) a (h) periodo de retorno de 475 años 

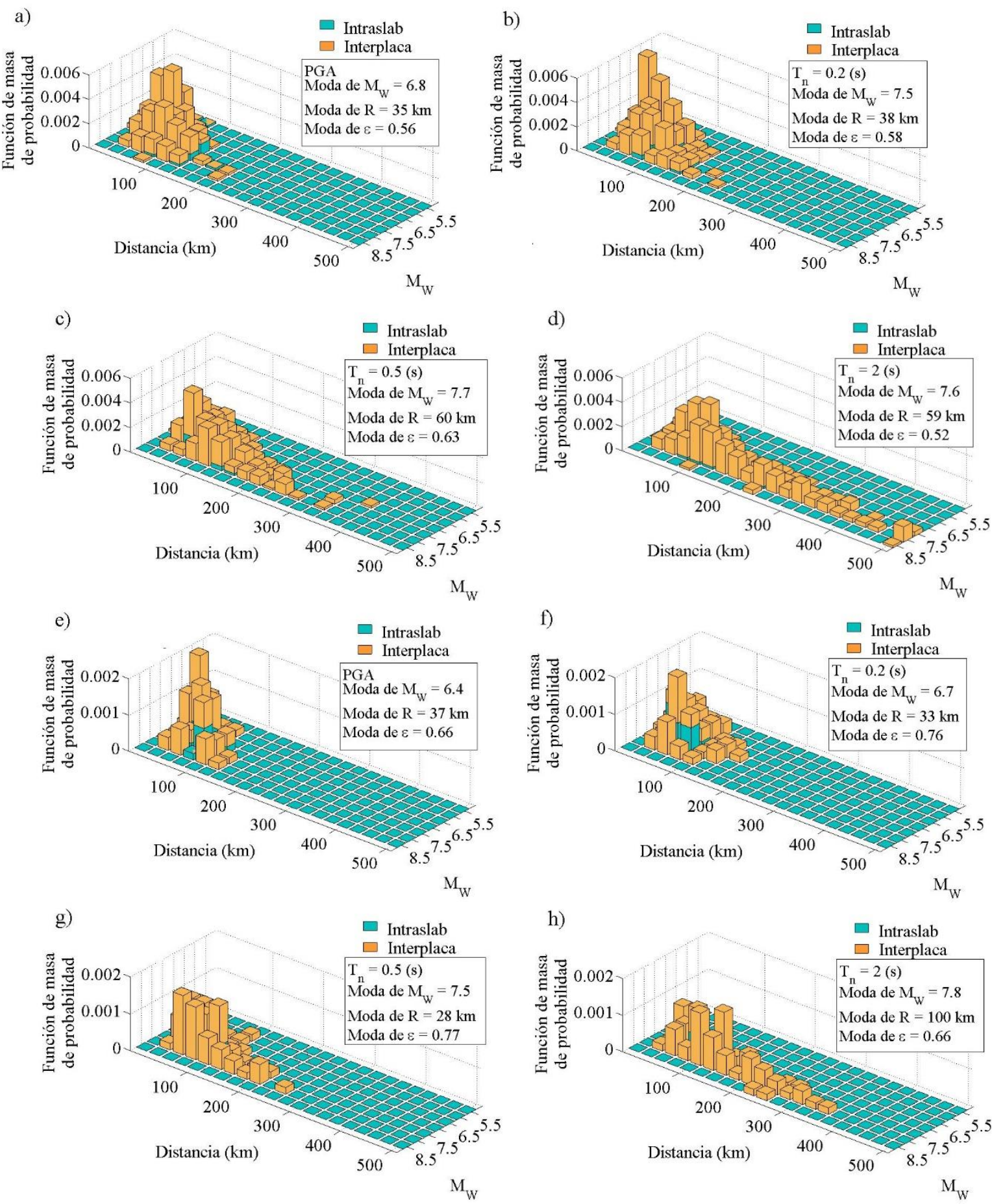

Figura 14. Desagregación sísmica para el Sitio IV (cerca de Salina Cruz): (a) a (d) periodo de retorno de 125 años; (e) a (h) periodo de retorno de 475 años

Dependiendo el sitio y el periodo de retorno, el intervalo de $M_{w}$ de los escenarios sísmicos identificados varía de 6.4 a 7.9, con valores de $R$ entre 18 y $270 \mathrm{~km}$, mientras que el intervalo de valores de $\varepsilon$ varía entre 0.10 y 0.86 . La variación de $\varepsilon$ indica que la desagregación del peligro sísmico es muy 
sensible al valor de $\varepsilon$ que se asocia con las relaciones de atenuación. Los resultados más detallados de la desagregación para el Sitio I se muestran en la figura 11, en donde se presentan las funciones de masa de probabilidad y los escenarios sísmicos identificados. Se observa en esta figura que para los periodos de retorno de 125 y 475 años, los eventos intraslab e interplaca contribuyen al peligro sísmico, aunque los eventos intraslab contribuyen con mayor frecuencia al peligro sísmico para la aceleración máxima del terreno y $T_{n}=0.2(\mathrm{~s})$, y en general los eventos interplaca contribuyen más al peligro sísmico para $T_{n}=0.5$ y 2 (s). Los parámetros modales de los escenarios sísmicos identificados, para un periodo de retorno de 125 años, son $(6.7,70,0.53),(7.5,67,0.22),(7.7,185,0.53),(7.8,175,0.48)$ para la aceleración máxima del terreno y las aceleraciones espectrales para $T_{n}=0.2,0.5$, y $2(\mathrm{~s})$, respectivamente. Los escenarios sísmicos identificados provienen de la zona de fuente sísmica de Profundidad intermedia Oeste Nueva (ver figura 1c) y de las costas de Guerrero y Michoacán (ver figuras 1b y 1c).

La figura 12 presenta los resultados de la desagregación sísmica para el Sitio II. La figura muestra que el peligro sísmico es dominado por eventos interplaca para los periodos de retorno de 125 y 475 años. Los eventos sísmicos identificados para un periodo de retorno de 125 años indican que existe un sismo importante, con $M_{w}$ entre a 7.5 y $7.7, R$ entre 30 y $45 \mathrm{~km}$, y $\varepsilon$ aproximadamente igual a 0.45 . Los valores específicos de $M_{w}, R$ y $\varepsilon$ dependen del valor de $T_{\mathrm{n}}$. Los eventos sísmicos identificados para un periodo de retorno de 475 años son similares en magnitud a aquéllos para 125 años, excepto que $R$ es menor y $\varepsilon$ aumenta. Una inspección de los resultados generados de la simulación indica que los eventos sísmicos identificados provienen de las zonas de fuentes sísmicas de Guerrero (ver figura 1b), como se esperaba.

La figura 13 presenta los resultados de la desagregación sísmica del Sitio III, en donde se observa que el peligro sísmico es dominado por eventos intraslab e interplaca para los periodos de retorno de $125 \mathrm{y}$ 475 años. Se observa una mayor contribución de los sismos intraslab al peligro sísmico para la aceleración máxima del terreno y para la aceleración espectral para $T_{n}=0.2(\mathrm{~s})$, mientras que para aceleraciones espectrales para $T_{n}=0.5$ y $2(\mathrm{~s})$, se observa una mayor contribución de los sismos interplaca. Los escenarios sísmicos identificados para un periodo de retorno de 125 años son $(6.8,79,0.10),(6.7,58$, $0.43),(7.7,113,0.74),(7.7,133,0.61)$ para la aceleración máxima del terreno y las aceleraciones espectrales para $T_{n}=0.2,0.5$, y $2(\mathrm{~s})$, respectivamente. Los escenarios sísmicos para un periodo de retorno de 475 años corresponden a eventos con $M_{w}$ entre 6.6 y $7.9, R$ entre 49 y $131 \mathrm{~km}$ y $\varepsilon$ entre 0.25 y 0.67 . Las zonas de fuentes sísmicas asociadas con los eventos identificados corresponden a la zona de fuente sísmica de Profundidad intermedia Centro (ver figura 1c) y las zonas de fuentes sísmicas de la costa de Oaxaca (ver figura 1b).

La figura 14 muestra los resultados de la desagregación sísmica del Sitio IV, donde el peligro sísmico es dominado por eventos interplaca para los periodos de retorno de 125 y 475 años. Los escenarios sísmicos identificados para un periodo de retorno de 125 años son $(6.8,35,0.56),(7.5,38$, $0.58),(7.7,60,0.63),(7.6,59,0.52)$ para la aceleración máxima del terreno y las aceleraciones espectrales para $T_{n}=0.2,0.5$, y $2(\mathrm{~s})$, respectivamente. Los eventos sísmicos para un periodo de retorno de 475 años comprenden valores de $M_{w}$ entre 6.4 y $7.8, R$ entre 33 y $100 \mathrm{~km}$ y $\varepsilon$ entre 0.66 y 0.77 . Los escenarios sísmicos identificados se asocian con las zonas de fuentes sísmicas de las costas de Oaxaca (ver figuras 1a y $1 b)$.

\section{CONCLUSIONES}

Se realizó la desagregación del peligro sísmico empleando un modelo de peligro sísmico aplicable a la región de la costa del Pacífico Mexicano y a la estación Ciudad Universitaria (estación CU) en la Ciudad de México. En el modelo se incluyen nuevas relaciones de atenuación desarrolladas para la estación CU. Con base en el modelo adoptado, se desarrollaron mapas de peligro sísmico para la región de la costa del 
Pacífico, y espectros de peligro uniforme (EPU) para diferentes periodos de retorno y algunos sitios seleccionados, incluyendo la estación CU. Para esta última se estudió la contribución de sismos interplaca e intraslab al espectro de peligro uniforme.

Las conclusiones principales se presentan a continuación:

- Se mostró que el uso de la relación de atenuación desarrollada empleando todos los registros obtenidos en la estación CU para los eventos intraslab moderados y grandes conlleva a un nivel de peligro sísmico considerable. Sin embargo, este nivel de peligro sísmico es reducido drásticamente si la relación de atenuación se desarrolla con base en los registros con excitaciones mayores que 10 (Gal). Una inspección adicional de las relaciones de atenuación indica que la basada en todos los registros es inadecuada debido a que provoca que los residuos se desvíen de la normalidad, y que la desviación estándar de los residuos sea mucho mayor que aquella obtenida de la relación de atenuación con base en registros de una red de estaciones más amplia. Lo anterior simplemente enfatiza la importancia de la selección de datos para desarrollar las relaciones de atenuación y la importancia de contar con una mayor cantidad de muestras.

- Aunque se puede desarrollar una relación de atenuación con base en los registros con excitaciones mayores que un límite inferior mayor que 10 (Gal), esto no fue considerado ya que se reduciría el número de acelerogramas que puede ser usado para desarrollar las relaciones de atenuación. La consideración del límite inferior de 10 (Gal) en el desarrollo de las relaciones de atenuación puede ser una suposición conservadora.

- Los sismos interplaca e intraslab contribuyen al peligro sísmico en la estación CU, en particular, el peligro sísmico es dominado por eventos intraslab para periodos de vibrar menores a 0.7 (s), mientras que los eventos interplaca dominan el peligro sísmico para periodos de vibrar mayores a 0.7 (s). Esta observación es confirmada por los resultados de desagregación del peligro sísmico.

- Los valores del espectro de diseño para la Ciudad de México son significativamente mayores a los valores del espectro de peligro uniforme para un periodo de retorno de 125 años. Se observa también que los valores del espectro de diseño para $T_{\mathrm{n}}$ entre 0.25 y 1.75 (s) son mayores a los valores del espectro de peligro uniforme para un periodo de retorno de 475 años.

- Los resultados de la desagregación, basados en el modelo de peligro sísmico adoptado, indican que ésta presenta escenarios sísmicos identificados en donde la contribución al peligro sísmico para un periodo de retorno de 125 o 475 años es dominada por un solo evento y en otros casos el peligro sísmico es debido a diferentes escenarios sísmicos y tipos de temblores. La identificación del tipo de temblor para escenarios sísmicos puede ser importante ya que las estadísticas de demanda de ductilidad pueden diferir para diferentes tipos de temblores. Los escenarios identificados dependen del periodo fundamental de vibrar de la estructura considerada. En general, como es de esperar, para los sitios en la región de la costa del Pacífico Mexicano, el peligro sísmico es dominado por sismos interplaca, mientras que para los sitios alejados de la costa del Pacífico, los sismos intraslab e interplaca contribuyen al peligro sísmico.

\section{RECONOCIMIENTOS}

El apoyo financiero recibido del Consejo Nacional de Ciencia y Tecnología (CONACyT) de México, The Natural Science and Engineering Research Council of Canada y del Instituto de Ingeniería de la 
UNAM es ampliamente agradecido. Agradecemos de manera especial los comentarios constructivos y sugerencias del Dr. J. A. Escobar y de dos revisores anónimos. También agradecemos a la Coordinación de Instrumentación Sísmica del IIUNAM por proveer algunos registros sísmicos empleados en este trabajo.

\section{REFERENCIAS}

Arroyo, D, D García, M Ordaz, M A Moray y S K Singh (2010), "Strong ground-motion relations for Mexican interplate earthquakes”, Journal of Seismology, vol. 14, pp. 769-785.

Atkinson G M y D M Boore (2003), "Empirical ground-motion relations for subduction-zone earthquakes and their application to Cascadia and other regions", Bulletin of the Seismic Society of America, vol. 93, pp. 1703-1729.

Atkinson G M (2006), "Single-station sigma", Bulletin of the Seismic Society of America, vol. 96, pp. 446-455.

Baker J W, C A Cornell y P Tothong (2005), "Deaggregation of seismic drift hazard", in Proc.9th International Conference on Structural Safety and Reliability (ICOSSAR05), Rome, Italy.

Bazzurro P y C A Cornell (1999), "Disaggregation of seismic hazard", Bulletin of the Seismic Society of America, vol. 89, pp. 501-520.

Boore D M, W B Joyner y T E Fumal (1997), "Equations for estimating horizontal response spectra and peak acceleration from western North America", Seismological Research Letters, vol.68, pp.128153. (for Erratum: see 2005, Seismological Research Letters, vol.76, pp. 368-369.

Chapman M C (1995), "A probabilistic approach to ground-motion selection for engineering design", Bulletin of the Seismic Society of America, vol. 85, pp. 937-942.

Cornell C A (1968), "Engineering seismic risk analysis", Bulletin of the Seismic Society of America, vol. 58, pp. 1583-1606.

Esteva L (1968), "Bases para la formulación de decisiones de diseño sísmico", Tesis Doctoral, Universidad Nacional Autónoma de México, México.

García D (2006), "Estimación de parámetros del movimiento fuerte del suelo para terremotos interplaca e intraslab en México Central", Tesis Doctoral, Universidad Complutense de Madrid, Madrid, España.

García D, S K Singh, M Herraiz, M Ordaz, y J P Pacheco (2005), "Inslab earthquakes of Central Mexico: Peak ground-motion parameters and response spectra", Bulletin of the Seismic Society of America, vol. 95 , pp. 2272-2282.

García-Pérez J, F Castellanos, O Díaz (2005), “Occupancy importance factor in earthquake engineering”, Engineering Structures, vol. 27, pp. 1625-1632.

García-Soto, A D, A Pozos-Estrada, H P Hong y R Gómez Martínez (2012), "Estimación del peligro sísmico debido a sismos interplaca e inslab y sus implicaciones en el diseño símico", Revista de Ingeniería Sísmica, No. 86, pp. 27 - 54.

Harmsen S, D Perkins y A Frankel (1999), "Deaggregation of probabilistic ground motions in the central and eastern United States", Bulletin of the Seismic Society of America, vol. 89, pp. 1-13. 
Hong H P (2012), "Peligro, riesgo, diseño sísmico óptimo", The Mexican Academy of Engineering, Mexico.

Hong H P y K Goda (2006), "A comparison of seismic hazard and risk deaggregation", Bulletin of Seismological Society of America", vol. 96(6), pp. 2021-2039.

Hong H P, A D Garcia-Soto y R Gomez (2010), "Impact of different earthquake types on the statistics of ductility demand", Journal of Structural Engineering, ASCE, vol. 136(7), pp. 770-780.

Hong H P, Y Zhang y K Goda (2009a), "Effect of the spatial correlation on the estimated ground motion prediction equations", Bulletin of the Seismological Society of America, vol. 99(2A), pp. 928-934.

Hong H P, A Pozos-Estrada y R Gomez (2009b), "Orientation effect on ground motion measure for Mexican subduction earthquakes", Earthquake Engineering and Engineering Vibration, vol. 8(1), pp. 1-16.

Hong H P, K Goda y A G Davenport (2006), "Seismic hazard analysis: a comparative study", Canadian Journal of Civil Engineering, vol. 33(9), pp. 1156-1171.

Hong H P y E Rosenblueth (1988), "The Mexico Earthquake of September 19, 1985 - Model for Generation of Subduction Earthquakes”, Earthquake Spectra, vol. 4(3), pp. 481 - 498.

Jaimes M A, y E Reinoso (2006), "Comparación de comportamiento de edificios en el Valle de México ante sismos de subducción y de falla normal”, Revista de Ingeniería Sísmica, No. 75, pp. 1-22.

Joyner W B y D M Boore (1993), "Methods for regression analysis of strong-motion data", Bulletin of the Seismic Society of America, vol. 83, pp. 469-487.

McGuire R K (1995), "Probabilistic seismic hazard analysis and design earthquakes: closing the loop", Bulletin of the Seismic Society of America, vol. 85, pp. 1275-1284.

McGuire R K (2004), "Seismic hazard and risk analysis", Oakland, Calif.: Earthquake Engineering Research Institute.

Montalvo-Arrieta J C (2002), "La respuesta sísmica del terreno firme en la ciudad de México. Observaciones y Modelos", Tesis Doctoral, Universidad Nacional Autónoma de México, Instituto de Geofísica, Posgrado en Ciencias de la Tierra.

Musson R M W (2000), “The use of Monte Carlo simulations for seismic hazard assessment in the UK". Annali di Geofisica, vol. 43, pp. 1-9.

Nishenko S P y S K Singh (1987), “The Acapulco-Ometepec, México earthquake of 1907-1982: Evidence for a variable recurrence history", Bulletin of the Seismic Society of America, vol. 77, pp. 13591367.

NTCDS (2004), "Normas Técnicas Complementarias para Diseño por Sismo", Reglamento de Construcciones para el Distrito Federal, Gaceta Oficial del Departamento del Distrito Federal, México, 6 de octubre de 2004.

Ordaz M y C Reyes (1999), "Earthquake hazard in Mexico City: Observations versus computations", Bulletin of the Seismic Society of America, vol. 89(5), pp. 1379-1383.

Ordaz M, E Miranda y J Avilés (2000), "Propuesta de espectros de diseño por sismo para el DF", Memorias del XII Congreso Nacional de Ingeniería Estructural, León, CD, noviembre.

Pozos-Estrada A, R Gomez y H P Hong (2008), "Seismic design: benefit/cost for overall service time versus per unit service time", 14 Word Conference on Earthquake Engineering, Beijing, China. 
Reinoso E y M A Jaimes (2009), "Criterios para obtener acelerogramas de diseño en sitios afectados por varias fuentes sísmicas usando como ejemplo el caso de terreno firme de la ciudad de México", Revista de Ingeniería Sísmica, No. 81, pp. 1 - 18.

Reyes C, E Miranda, M Ordaz y R Meli (2002), "Estimación de espectros de aceleraciones correspondientes a diferentes periodos de retorno para las distintas zonas sísmicas de la ciudad de México", Revista de Ingeniería Sísmica, No. 66, pp. 95 - 121.

Rosenblueth E y M Ordaz (1987), "Use of seismic data from similar regions", Earthquake Engineering \&. Structural Dynamics, vol. 15, pp. 619-634.

Rosenblueth E y J M Jara (1991), "Constant versus time dependent seismic design coefficients", Proceedings of the 3rd IFIP WG 7.5 Conference on Reliability and Optimization of Structural Systems '90, Springer-Verlag, Berlin, Germany, vol. 1, pp. 315-327.

Rosenblueth E, M Ordaz, J F Sánchez-Sesma y S K Singh (1989), “The Mexico Earthquake of September 19, 1985 - Design Spectra for Mexico's Federal District”, Earthquake Spectra, vol. 5(1), pp. 273 291.

Singh S K, M Rodriguez y L Esteva (1983), "Statistics of small earthquakes and frequency of occurrence of large earthquakes along the Mexican subduction zone", Bulletin of the Seismic Society of America, vol. 73, pp. 1779-1796.

Singh S K, M Ordaz, X Pérez-Campos y A Iglesias (2013), "Intraslab versus Interplate Earthquakes as Recorded in Mexico City: Implications for Seismic Hazard", Earthquake Spectra, doi: http://dx.doi.org/10.1193/110612EQS324M. 